\title{
Local, nonlocal quantumness and information theoretic measures
}

\author{
Pankaj Agrawal,,, Sk Sazim, ${ }^{1, \dagger}$ Indranil Chakrabarty, ${ }^{2}$ and Arun K. Pati ${ }^{3}$ \\ ${ }^{1}$ Institute of Physics, Sainik School Post, Bhubaneswar-751005, Orissa, India. \\ ${ }^{2}$ Center for Security, Theory \& Algorithmic Research, International Institute for Information Technology, Hyderabad, India \\ ${ }^{3}$ Harish-Chandra Research Institute, Chhatnag Road, Jhunsi, Allahabad 211 019, India
}

(Dated: July 16, 2021)

\begin{abstract}
It has been suggested that there may exist quantum correlations that go beyond entanglement. The existence of such correlations can be revealed by information theoretic quantities such as quantum discord, but not by the conventional measures of entanglement. We argue that a state displays quantumness that can be of local and nonlocal origin. Information theoretic measures not only characterize the nonlocal quantumness but also the local quantumness, such as the "local superposition". This can be a reason why such measures are nonzero when there is no entanglement. We consider a generalized version of the Werner state to demonstrate the interplay of local quantumness, nonlocal quantumness, and classical mixedness of a state.
\end{abstract}

\section{INTRODUCTION}

One of the basic problems in quantum physics is to understand the nature of correlations present between different particles in a composite system. The existence of nonfactorizable states play important role in the existence of many exotic features of quantum information theory. These features, more specifically the advantages, include phenomena like quantum cryptography [1], quantum computation [2, 3], quantum imaging [4], quantum phase transition [5], quantum biology [6] and many more [7]. Therefore, it is important to study and understand the nature of correlations present in various quantum systems. In the last decade, various measures of correlations [8-13] have been introduced. It is believed that none of these measures can alone be sufficient to manifest all the facets of quantum correlations. However, each of these measures unveils some aspects of quantum correlations.

Entanglement is the key concept which alters the notion of reality in the microscopic system. Not only that, it is also responsible for the metamorphosis of the meaning of correlation as we move from classical systems to quantum systems. Various studies have been conducted regarding the detection as well as quantification of entanglement [14-16]. Multitudinous techniques and measures have been introduced for the detection and quantification of entanglement. For pure states the situation is quite comprehensible as entanglement tells all about the correlation present. However, the situation is not so clear in the case of mixed states. In the case of mixed states because of certain issues, researchers begin to have a hunch that there may be something beyond the entanglement that actually quantifies the amount of correlation present in the system. Recently, many measures have been proposed to quantify the amount of correlation present in a mixed state. These measures have a unique feature that all of them seem to predict the presence of quantum correlation beyond the domain of entanglement. However, the nature of these correlation, as one goes

\footnotetext{
*agrawal@iopb.res.in

† ssazim@iopb.res.in
}

beyond the entanglement, is far from understood. There have been many speculations, but still there is no universal way by which one can determine the total correlation present in the system [17].

There are two aspects of the quantum mechanical formalism that play important role in quantum information processing. The one aspect may be referred to as nonlocal quantumness. This is due to nonlocal superposition. This nonlocal superposition leads to entanglement. We will call the other aspect as local quantumness. The local quantumness appears due to local superposition of the states. Every extant correlation measures which is non-zero for separable states will show such quantumness. For the sake of illustration and simplicity, we will focus here on the information theoretic measure quantum discord.

Of the late, quantum discord have been accentuated in many works [9-11]. This is an important information theoretic measure based on the mutual information. It is the difference between the total correlation and the classical correlation present in the system. Here in this work, we demonstrate that such quantities probe not only nonlocal quantumness but also local quantumness. That is the prime reason why such measures are non-zero for mixed states even when there is no entanglement present in the system.

The organization of the work is as follows. In the section II, we discuss the notion of quantum covariance to characterize the correlations. In the section III, we give a brief introduction to the quantum discord. In the section IV, we discuss the phenomenon of local and nonlocal quantumness. In the section $\mathrm{V}$, we consider few states to exemplify the difference between the classical and separable states in the context of local and nonlocal quantumness. In the section VI, we introduce the parametric representation of local and nonlocal quantumness and show that the discord function depends on both of them. Finally, we conclude in the last section. 


\section{QUANTUM COVARIANCE}

The covariance for a bipartite state $\rho_{X Y}$ is defined as

$$
\begin{array}{r}
\operatorname{Cov}\left(\rho_{X Y}, \mathcal{O}_{X}, \mathcal{O}_{Y}\right)=\operatorname{Tr}_{X Y}\left(\rho_{\mathrm{XY}} \mathcal{O}_{\mathrm{X}} \mathcal{O}_{\mathrm{Y}}\right)- \\
\operatorname{Tr}_{\mathrm{X}}\left(\rho_{\mathrm{X}} \mathcal{O}_{\mathrm{X}}\right) \operatorname{Tr}_{\mathrm{Y}}\left(\rho_{\mathrm{Y}} \mathcal{O}_{\mathrm{Y}}\right),
\end{array}
$$

where $\mathcal{O}_{X}$ and $\mathcal{O}_{Y}$ are observables acting on the part $X$ and $Y$ respectively. Unlike its classical counterpart, this covariance is not a measure of quantum entanglement (or quantum correlations). However, we can use it to detect quantum correlations. (See also the discussion below about nonlocal quantumness.) Using the intuitive meaning of quantum correlation, one can argue that a bipartite pure state has no quantum correlations, if the covariance vanishes for any two arbitrary observables $X$ and $Y$. Clearly, covariance vanishes for the product states $\rho_{X Y}=\rho_{X} \otimes \rho_{Y}$. Here $\rho_{X}$ and $\rho_{Y}$ are reduced density matrices. For a mixed state, one can minimize the magnitude of quantum covariance over all possible decompositions. We can then define covariance for the system with the density matrix $\rho_{X Y}=\sum_{i} p_{i} \rho_{X Y}^{i}$ as

$$
\Lambda\left(\rho_{X Y}\right)=\min \sum_{i} p_{i}\left|\operatorname{Cov}\left(\rho_{X Y}^{i}, \mathcal{O}_{X}, \mathcal{O}_{Y}\right)\right|
$$

To avoid the negative value of covariance, we have considered its magnitude. In case the $\Lambda\left(\rho_{X Y}, \mathcal{O}_{X}, \mathcal{O}_{Y}\right)$ is non-zero, then the state will have quantum correlations.

Lemma 1. For all bipartite two-qubit separable states, $\Lambda\left(\rho_{X Y}\right)=0$.

Proof: Here we can use the fact that (a) all the separable states can be decomposed in terms of product states and (b) for product states $\Lambda=0$.

Hence the 'Lemma 1' is important to identify bipartite correlated states.

\section{QUANTUM DISCORD}

The quantum discord was introduced by Olivier and Zurek (2002) [9] as a measure of the "quantumness of correlations". It is defined in terms of the mutual information. Classically, the mutual information is a measure of common information in two random variables. Therefore, it was natural to generalize it to the quantum domain and express quantum correlation in terms of this object. However, the definition of the mutual information in quantum domain is not straightforward. This is because there are more than one classical expressions to define the mutual information. These different expressions admit different generalizations. Discord uses this difference to characterize the quantum correlations. Classically, one can write the mutual information in two alternate ways, $I(X: Y)=H(X)-H(X \mid Y)$, and $J(X$ : $Y)=H(X)+H(Y)-H(X, Y)$. Here $H(X), H(X, Y)$ and $H(X \mid Y)$ are the entropy, joint entropy, and conditional entropy for the random variables $X$ and $Y$. The Joint entropy and conditional entropy are related by the chain rule, $H(X \mid Y)=H(X, Y)-H(Y)$.

These expressions for the entropies can be generalized to the quantum domain by substituting random variables by density matrices and Shannon entropies by von Neumann entropies. For example, $H(X) \rightarrow H\left(\rho_{X}\right)=-\operatorname{Tr}[\rho \log (\rho)]$. The generalization of the mutual information will also involve the generalization of the conditional entropy. We use the generalization as suggest in the Ref [9]. Using this generalization to the quantum domain, we obtain $I(X: Y)=H(X)-$ $H\left(X \mid\left\{\pi_{i}^{Y}\right\}\right)$, where $H\left(X \mid\left\{\pi_{j}^{Y}\right\}\right)=\sum_{j} p_{j} H\left(\rho_{X \mid \pi_{j}^{Y}}\right)$ with $\rho_{X \mid \pi_{j}^{Y}}=\frac{\pi_{j}^{Y} \rho_{X Y} \pi_{j}^{Y}}{\operatorname{Tr}\left(\pi_{j}^{Y} \rho_{X Y}\right)}$ (where $p_{j}$ is the probability of obtaining the $j$ th outcome). Here, $H\left(X \mid\left\{\pi_{j}^{Y}\right\}\right)$ is the Von Neumann entropy of the qubit $X$, when the projective measurement is done on $Y$. The quantum discord is then defined as, $D(X: Y)=J-I=H(Y)-H(X, Y)+H\left(X \mid\left\{\pi_{j}^{Y}\right\}\right)$. This is to be minimized over the set of all one dimensional projectors $\left\{\pi_{i}^{Y}\right\}$. We shall call $D(X: Y)$ as discord function and its minimum value as the quantum discord. It is evident that the discord function is not symmetric in $X$ and $Y$. In the above definition, we are making a measurement on the system $Y$. Let us call it $Y$-discord. Similarly, we can define $X$-discord, when the measurement is made on the system $X, D(Y: X)=H(X)-H(X, Y)+H\left(Y \mid\left\{\pi_{j}^{X}\right\}\right)$. Here $H\left(Y \mid\left\{\pi_{j}^{X}\right\}\right.$ is defined in the same way as $H\left(X \mid\left\{\pi_{j}^{Y}\right\}\right)$.

For a bipartite state, $X$-discord and $Y$-discord may have different values. They will have identical values when the state is symmetric in $X$ and $Y$. But, they are always nonnegative. When one of the discord is zero, then the state would be separable. However it still may not be completely classical state and may exhibit quantum behaviour. For the state to be completely classical, both discords must vanish. As we shall see below, there exist states for which only one of these discords is zero. Therefore, for the complete characterization of the quantumness, one should know both discords. For our convenience, we define a vector quantity, $\vec{\delta}$ which contains both discord as,

$$
\vec{\delta}\left(\rho_{X Y}\right)=\{\delta(X: Y), \delta(Y: X)\}
$$

where $\delta(X: Y)$ and $\delta(Y: X)$ are the $X$-discord and $Y$ discord respectively after minimization over measurement parameters.

Observation 1. A two-qubit state is either classically correlated or is a product state iff $\vec{\delta}=\overrightarrow{0}$.

In the literature, there exit witness operators for discord (cf. [13]) but we will not discuss them here. Observation 1 is enough for our analysis and it also gives us information about the structure of the states. 


\section{QUANTUMNESS - LOCAL AND NONLOCAL}

A state of a bipartite quantum system may exhibit nonclassical behaviour due to either the local superposition ("local quantumness") or due to the nonlocal superposition, i.e. entanglement, ("nonlocal quantumness"). Usually, one is more concerned about the entanglement and its characterization and quantification - in part due to its mysterious nature and to use it as a resource. However, local quantumness can also be important if we can exploit the superposition as a resource in general. It is the superposition, local or nonlocal, that gives advantage in many quantum information processing protocols.

In the case of quantum discord, therefore we have $D(X$ : $Y)=D\left(\varphi_{L}(X: Y), \varphi_{N L}(X: Y)\right)$ where $\varphi_{L}(X: Y)$ characterizes the local quantumness, and $\varphi_{N L}(X: Y)$ characterizes the nonlocal quantumness of the state. We don't know yet if $D(X: Y)=D\left(\varphi_{L}(X: Y)+D\left(\varphi_{N L}(X: Y)\right.\right.$. Their properties are -1$)$ both $\varphi_{L}(X: Y)$ and $\varphi_{N L}(X: Y)$ are invariant under local unitary operation; 2) $\varphi_{L}(X: Y)$ may increase under local operations, but $\varphi_{N L}(X: Y)$ would not; 3$)$ under global operations, $D(X: Y)$ may increase or decrease; 4) if the state is separable then $D(X: Y)=D\left(\varphi_{L}(X: Y)\right)$ and $D(X: Y)=D\left(\varphi_{L}(X: Y), \varphi_{N L}(X: Y)\right)$ for an entangled state. We now discuss these features of a quantum state in a bit more detail.

\section{A. Local Quantumness}

A bipartite separable quantum state may not have entanglement, but it is a quantum state and can exhibit quantum features. This quantum feature may be called as the local quantumness. What we mean by local quantumness can be seen by following three examples. Consider the density operators

$$
\begin{aligned}
& \rho_{a}=|++\rangle\langle++|, \\
& \rho_{b}=p|++\rangle\langle++|+(1-p)|--\rangle\langle--|, \\
& \rho_{c}=q|++\rangle\langle++|+(1-q)| 00\rangle\langle 00|,
\end{aligned}
$$

where $| \pm\rangle=\frac{1}{\sqrt{2}}(|0\rangle \pm|1\rangle)$. The state $\rho_{a}$ is a pure quantum state with no entanglement. Now one can argue that this state shows local quantumness. However, this local quantumness can be masked in the case of a pure product state. If we make a local measurement on the particle ' $A$ ' in Hadamard basis, we will get the particle in the state $|+\rangle$ with unit probability and the state would not change after the measurement. So, the local quantumness may not apparent. However, if we make a measurement in the computational basis $\{|0\rangle,|1\rangle\}$, then the particle ' $A$ ' can be found in any of the computational basis state with equal probability and the state would change after the measurement. This can be easily seen if we think of the state as a local superposition of the computational basis states. The state $\rho_{b}$ is what is known as classical mixed state. Its behavior will be similar to $\rho_{a}$ with respect to the measurements. We can mask its local quantumness. The state $\rho_{c}$ is also a separable state. However, in this state we cannot mask the local quantumness, irrespective of the measurement basis. This is because one particle state is not orthogonal and the state in one of mixture component can be written in terms of the superposition of the state in the other component and the rest of the measurement basis. Therefore, irrespective of the measurement basis, local quantumness (local superposition) cannot be hidden. So, we see that a separable state which is not completely classical, will have local superposition which can be exploited. This is what has been showing up as a resource in the case of, eg, the model deterministic quantum computational with one quantum bit (DQC1) [3].

Lemma 2. A two qubit state $\rho_{X Y}$ has only local quantumness iff $\Lambda\left(\rho_{X Y}\right)=0$ and $\vec{\delta}\left(\rho_{X Y}\right) \neq \overrightarrow{0}$.

Proof: The proof follows from the observations (a) for all twoqubit separable states $\Lambda\left(\rho_{A B}\right)=0$ and, (b) only for product states, or, classical states $\vec{\delta}\left(\rho_{X Y}\right)=\overrightarrow{0}$.

\section{Local noise can enhance Discord}

Since discord probes also local quantumness, therefore it can even increase by local operations. However, the local operation should be such that it changes the relative local quantumness of the mixture components. Quantum noise can be a good candidate for such a local operation. However standard local noise such as bit flip and phase flip noise cannot change discord, because no relative local superposition is introduced. In Ref [18], a set of Krass operators are given which can convert a classical mixed state, like $\rho_{1}$, given below in Eq. (5), to a classical-quantum mixed state, like $\rho_{2}$ or $\rho_{3}$, given below. This local noise can convert one separable state to another separable state, but not to an entangled state. This noise is only changing the local quantumness properties of a bipartite state.

\section{B. Nonlocal Quantumness}

In this paper, we shall mean the existence of quantum correlations in a state as equivalent to the state showing "nonlocal quantumness". It will also be synonymous with the existence of entanglement. If there is a system made of two subsystems, and there are quantum correlations, then the properties of the one subsystem, say $A$, would depend on the properties of the other subsystem, say $B$. The states of the subsystems are interdependent. This is the intuitive meaning of correlations. One can give a criteria for a pure bipartite state to possess quantum correlations. This criteria can then be generalized to a mixed state. This has been discussed in the section II.

Observation 2. A two qubit state $\rho_{X Y}$ has nonlocal quantumness iff $\Lambda\left(\rho_{X Y}\right) \neq 0$.

This just follows from the Lemma 1 .

We have discussed above the quantumness of a state goes beyond entanglement. We suggest that discord characterizes the quantumness of a state. This quantumness has both local 
and nonlocal components. A separable state can have local quantumness, but no nonlocal quantumness. An individual system may also show quantum, i.e., non-classical behaviour. So quantum behaviour of any system encompasses quantumness due to correlation and quantumness of an individual system in the absence of correlation. Essence of the local quantumness is due to the superposition property of the state of a subsystem of a composite system. We can visualize the classification of bipartite states as in Fig 1.

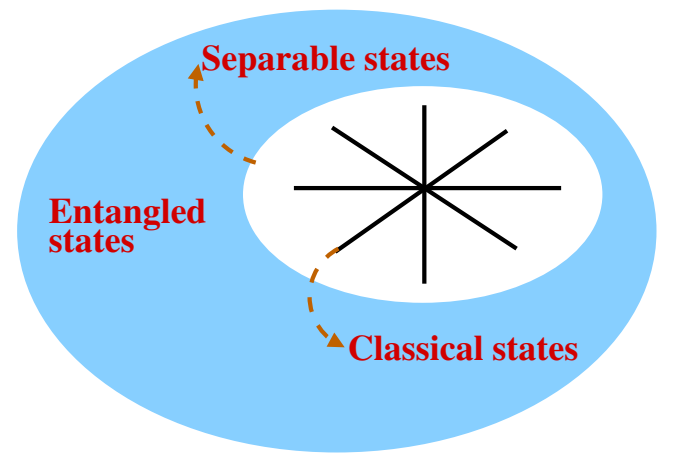

FIG. 1: (Color online) The large ellipse represents all two-qubit states (cf. [19] ). The small ellipse represents all separable states (i.e., $\Lambda=0$ ). The lines represent set of product states (end points of the lines) and classical states (in different basis). The point where the lines meet is the maximally mixed state. The outer annular space contains all entangled or, nonlocal states (i.e., $\Lambda \neq 0 \& \vec{\delta} \neq \overrightarrow{0}$ ), inner ellipse (except the lines) contains all separable states with local quantumness (i.e., $\Lambda=0 \& \vec{\delta} \neq \overrightarrow{0}$ ) and line depicts all product states and classical states $(\Lambda=0 \& \vec{\delta}=\overrightarrow{0})$.

This is in accordance with the fact that while the density operator $\rho_{1}$ represents a classical mixture, i.e., a mixture of orthogonal states, the density mixture $\rho_{4}$ represents a mixture of non-orthogonal states. In the case of $\rho_{4}$, unlike $\rho_{1}$, states in one of the component, $|+\rangle$ is a linear superposition of the computational basis states $\{|0\rangle,|1\rangle\}$. This is the case of local superposition. Therefore, the discord is non-zero for $\rho_{4}$ because it also probes local quantumness (apart from nonlocal quantumness due to entanglement). One can say that a

\section{SIMPLE EXAMPLES}

\section{A. Separable and Classical States}

In order to exemplify our argument, we consider separable mixtures and examine the discord function for them. In the mixed state domain, a state is said to be separable if it can be expressed as convex combination of product states. So, in principle a product state is a separable state while the converse is not always true. Therefore, separable states do not possess entanglement. However, as is known, not all separable states have zero discord. As a paradigm, we start with following mixed states,

$$
\begin{aligned}
& \rho_{1}=p|00\rangle\langle 00|+(1-p)| 11\rangle\langle 11|, \\
& \rho_{2}=p|++\rangle\langle++|+(1-p)| 0-\rangle\langle 0-|, \\
& \rho_{3}=p|++\rangle\langle++|+(1-p)|-0\rangle\langle-0|, \\
& \rho_{4}=p|++\rangle\langle++|+(1-p)| 00\rangle\langle 00|,
\end{aligned}
$$

where $| \pm\rangle=\frac{1}{\sqrt{2}}(|0\rangle \pm|1\rangle)$ are the Hadamard states. These density matrices represent four different categories of separable states. Neither of these states have entanglement. However, these states differ in important ways. $\rho_{1}$ belongs to the category of completely classical states. $\rho_{2}$ and $\rho_{3}$ are not completely classical, because, in the mixture, the states of only one of the particles are orthogonal. In the case of $\rho_{1}$, both $X$-discord and $Y$-discord are zero. For $\rho_{2}, X$-discord is zero, while for $\rho_{3}, Y$-discord is zero. For $\rho_{4}$, both discords are nonzero. If we make a measurement in computational basis, then the discord function is nonzero for $\rho_{1}$ and $\rho_{2}$. But we have to minimize the discord function to obtain the discord, the discord is zero for $\rho_{1}$, but not for $\rho_{2}$. For $\rho_{1}$, the discord function is zero in the Hadamard basis. This is the basis formed out of the states, of which the $\rho_{1}$ is a mixture. In this basis conditional entropy is zero, while entropies of the individual and composite system cancel. These facts are illustrated in Figs 1 and 2, where discord functions are plotted as a function of the angle $\theta$ that characterizes the measurement basis [11]. In these plots, $D X=D(Y: X)$ and $D Y=D(X: Y)$.

mixture of non-orthogonal separable state has local quantumness, i.e., local superposition, which cannot be washed away by writing down another decomposition of the density matrix.

\section{B. Werner State}

In this subsection, we show the importance of local quantumness for non vanishing value of the quantum discord with 


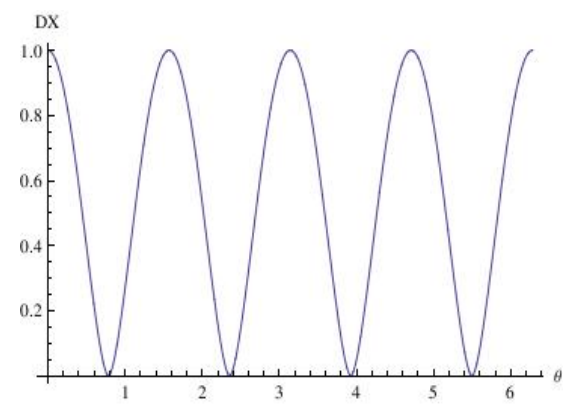

$(i)$

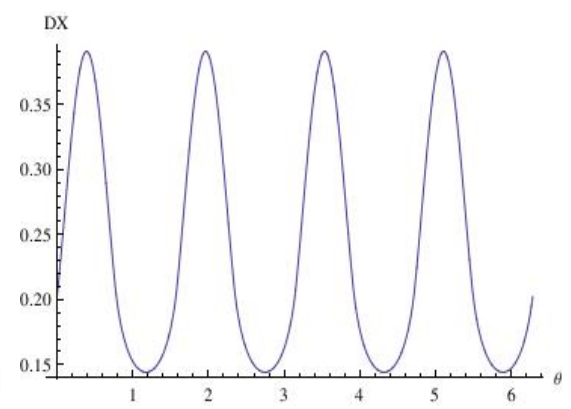

(ii)

FIG. 2: Dependence of $X$-Discord function on measurement basis with classical mixing parameter $p=0.5$ for (i) $\rho_{1}$ and (ii) $\rho_{4}$.

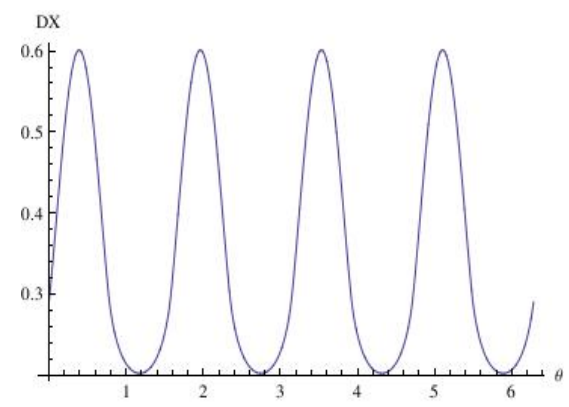

(i)

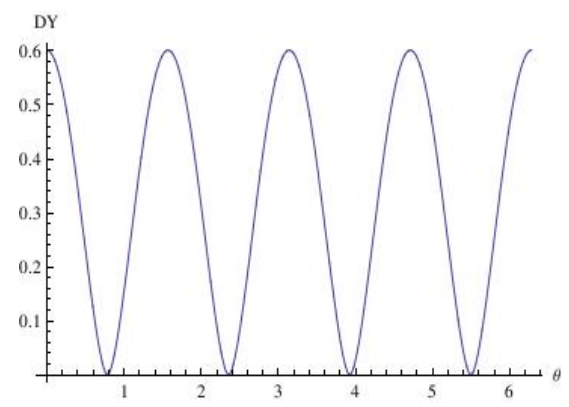

(ii)

FIG. 3: Dependence of (i) $X$-Discord and (ii) $Y$-Discord functions on measurement basis with classical mixing parameter $p=$ 0.5 for $\rho_{2}$. For $\rho_{3}$ the $X$-Discord and $Y$-Discord are interchanged.

the aid of the Werner state. This state is given by

$$
\rho_{\mathrm{w}}=(1-p) \frac{\mathbb{I}}{4}+p\left|\Phi^{+}\right\rangle\left\langle\Phi^{+}\right|,
$$

where $\left|\Phi^{+}\right\rangle=\frac{1}{\sqrt{2}}(|00\rangle+|11\rangle)$ is a Bell state, $\mathbb{I}$ is the identity operator and $p$ is the classical mixing parameter. Naively, one may think that this state is not separable and has quan-

At this point we ask this question: Does it necessarily mean that Werner state has quantum correlations that, in some sense, go beyond entanglement? We claim that the answer to this question is no. Our argument is that one can always rewrite Werner state in such a way that this state is a valid mixture of non-orthogonal states whenever $p<\frac{1}{3}$ [12]. Therefore the discord is nothing but just revealing the local quantumness. tum correlations for all values of classical mixing parameter $p$. However, it is known that this state is not entangled when $p<\frac{1}{3}$ (using Peres-Horodecki criterion [20], e. g.). It is also known that this state (pseudo pure state) is useful for information processing. If we look at the plot of the discord and the concurrence in the Fig 3, we see that concurrence is zero, when the state is not entangled, but the discord is non-zero.

Rewriting the Werner state in that form, we have

$$
\begin{aligned}
& \rho_{\mathrm{w}}=(1-3 p) \frac{\mathbb{I}}{4}+\frac{p}{2}(|++\rangle\langle++|+|--\rangle\langle--| \\
& |00\rangle\langle 00|+| 11\rangle\langle 11|+| \tilde{+} \tilde{-}\rangle\langle\tilde{+} \tilde{-}|+| \tilde{-} \tilde{+} \mid\rangle\langle|\tilde{-} \tilde{+}|),
\end{aligned}
$$

where $|\tilde{ \pm}\rangle=\frac{1}{\sqrt{2}}(|0\rangle \pm i|1\rangle)$. This is a valid density operator when $p \leq \frac{1}{3}$. This is precisely the region of $p$, where Werner state is not entangled. Since $\langle+\mid 0\rangle \neq 0$ and $\langle+\mid \tilde{+}\rangle \neq 0$, this 


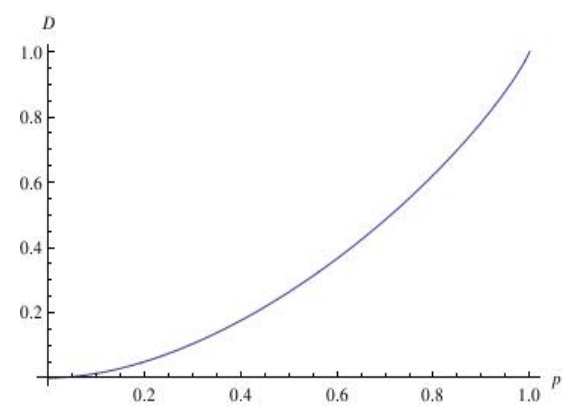

(i)

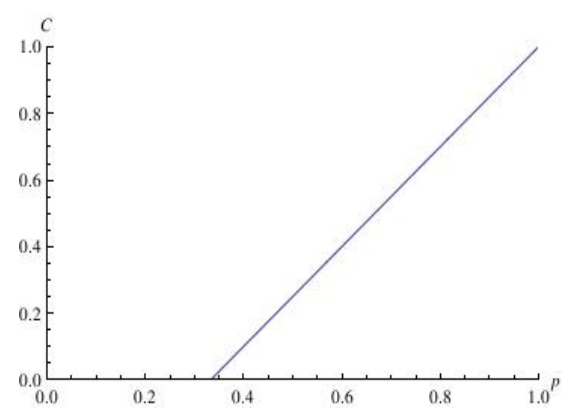

(ii)

FIG. 4: (i) Quantum discord (D) and (ii) Concurrence (C) for the Werner State as a function of the classical mixing parameter $p$.

state is a mixture of separable non-orthogonal states; so it is expected to have non-zero discord due to local quantumness.

\section{GENERALIZED WERNER STATE: A COMPARATIVE ANALYSIS OF ENTANGLEMENT AND DISCORD}

In this section we generalize the Werner state to investigate the interdependence of local quantumness, nonlocal quantumness and classical mixedness by parametrization of each of these quantities. The major thrust of our claim lies in this part where we are able to see that the measures of entanglement like concurrence are independent of local quantumness, where as discord is a function of all these quantities. The generalized Werner state is defined as

$$
\rho_{\mathrm{GW}}=(1-p) \frac{\mathbb{I}}{4}+p\left|\Phi_{n k}^{+}\right\rangle\left\langle\Phi_{n k}^{+}\right|,
$$

where, $\left|\Phi_{n k}^{+}\right\rangle=N_{n k}\left(|+\rangle_{n}|+\rangle_{n}+k|-\rangle_{n}|-\rangle_{n}\right),|+\rangle_{n}=$ $N(|0\rangle+n|1\rangle) \quad|-\rangle_{n}=N\left(-n^{*}|0\rangle+|1\rangle\right)$. Here $N_{n k}$ and $N$ are normalization constants. We can think of $n$ as a local superposition parameter; $k$ as a nonlocal superposition parameter and $p$ as the classical mixing parameter. We note that this state becomes a separable state as $k \rightarrow 0$. Furthermore, there is no local superposition as $n \rightarrow 0$. To study the behavior of the state with respect to these parameters, we compute concurrence and discord for this state. To see how the discord and concurrence change as we vary $p, n$ and $k$, in the following figures, we have plotted these functions. In the Fig 4, we have plotted concurrence for two different values of $p$ as a function of the parameters $n$ and $k$. We observe that concurrence is independent of the local superposition parameter $n$. It is important because discord depends on $n$. It is expected that measures of entanglement are independent of local superposition parameter $(n)$, while the measures of correlations which claim to go beyond entanglement will depend on it. Coming back to these, we see that concurrence vanishes when mixing is small and the state is not entangled. It is also noteworthy to see that larger the value of $p$, larger is the concurrence. Concurrence also vanishes when nonlocal superposition parameter is small. In other words, if one is small then other has to be large for the state to be entangled. In fact, we find that this generalized Werner state is entangled, i.e., the concurrence is non-zero when

$$
p>\frac{\left(1+k^{2}\right)}{\left(1+k^{2}+4 k\right)} .
$$

This requirement is independent of $n$. And it reduces to familiar condition $p>\frac{1}{3}$ for the Werner state $(n=0, k=1)$ in order that it is entangled.

Let us now see how discord varies with respect to changes in $p, n$ and $k$. Similar to the concurrence, the discord is plotted in Figs 5 and 6. With the increase of the value of mixing parameter, the value of discord increases. Even for very small values of mixing, when there is expected to be no entanglement, the discord is non-zero. When there is no local superposition, i.e., $n=0$, the discord value increases as mixing becomes stronger. The value also increases, as the value of nonlocal parameter $k$ increases, i.e., entangled component of the mixture becomes more entangled, as expected. When there is no nonlocal superposition, i. e. $k=0$, and the mixture is separable, the discord is non-zero. Its value increases, as the mixing parameter increases, or local quantumness becomes stronger. Here important point is that the concurrence is independent of the local superposition parameter $n$, while the discord increases with an increase in the value of $n$. 


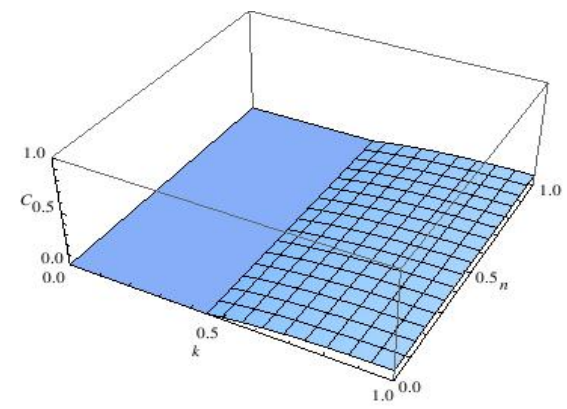

(i)

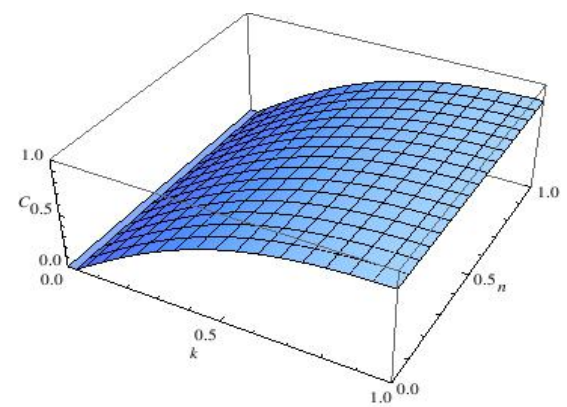

(ii)

FIG. 5: Variation of concurrence (C) for the Generalized Werner State with local superposition parameter $n$ and nonlocal superposition parameter $k$ for the two values of classical mixing parameter (i) for $p=0.4$ and (ii) $p=0.9$.
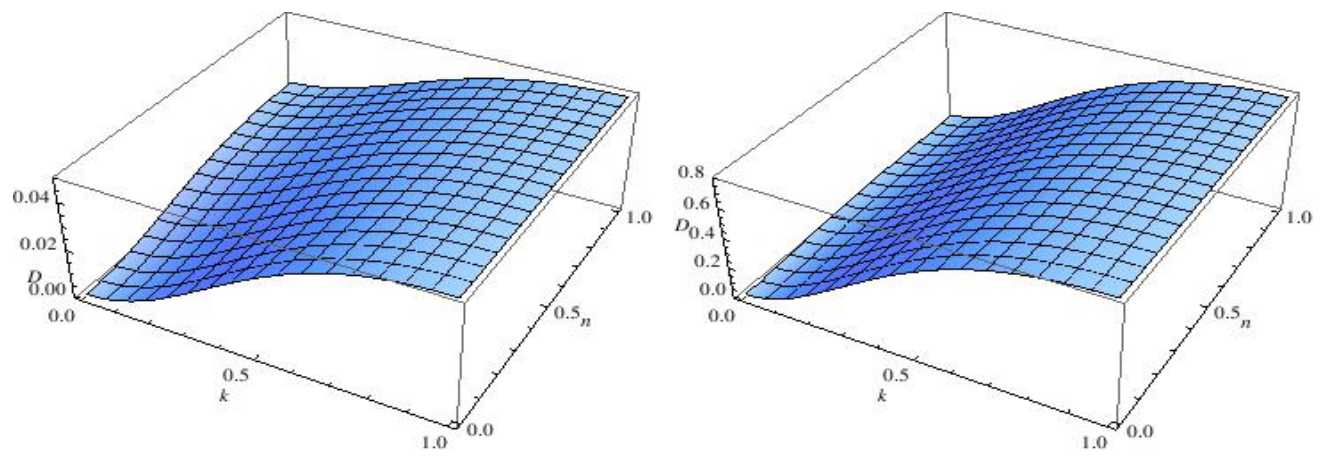

FIG. 6: Variation of quantum discord (D) for the Generalized Werner State with local superposition parameter $n$ and nonlocal superposition parameter $k$ for the two values of classical mixing parameter (i) for $p=0.2$ and (ii) $p=0.9$.

\section{CONCLUSION}

We have proposed that quantum discord (and other similar measures) as a measure of quantum correlations for a bipartite system contains both the local and the nonlocal quantumness. A quantum states with nonzero value of discord does not mean existence of quantum correlations beyond entanglement. In the absence of entanglement, there can be local quantumness that can make the discord nonzero. We have illustrated our proposal using a generalized Werner state to demonstrate the interplay of local quantumness, nonlocal quantumness, and classical mixedness by computing concurrence and quantum discord. To characterize the quantumness of a state, one also needs to compute both X-discord and Y-discord. Both discords have to be zero to mask the local quantumness.

We hope the present findings will help in understanding the nature of quantumness that goes beyond entanglement.

Acknowledgement: P. Agrawal thank the organizers of the event Quantum Discord Workshop, 2012, CQT, Singapore for providing the platform to present this work and having fruitful discussions with the experts in the fields.
[1] N. Gisin et al., Rev. Mod. Phys., 74, 145, 2002; A. Ekert, Phys. Rev. Lett. 67, 661 (1991); C. H. Bennett and G. Brassard, Proceedings of IEEE International Conference on Computers, System and Signal Processing, Bangalore, India, pp.175-179 (1984).

[2] See, eg, H. J. Briegel et al, Nat. Phys. 5, 19 (2009); R. Chaves and F. de Melo, Phys. Rev. A 84, 022324 (2011).

[3] See, eg, A. Datta, A. Shaji, C. M. Caves, Phys. Rev. Lett. 100, 050502 (2008); E. Knill and R. Laflamme, Phys. Rev. Lett. 81, 5672 (1998).

[4] See, eg, L. A. Lugiato, A. Gatti and E. Brambilla, J. Opt. B: Quant. Semiclass. Opt. 4 S176 (2002); T. Ono, R. Okamoto, 


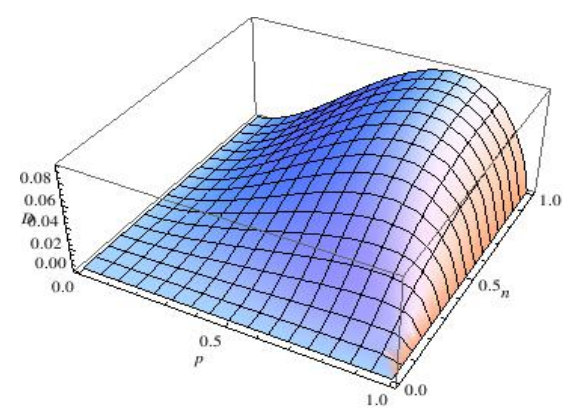

$(i)$

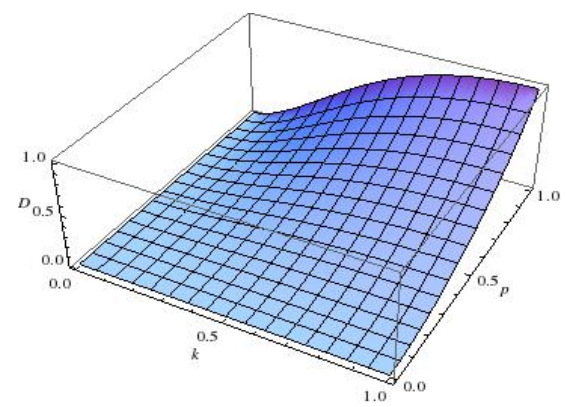

(ii)

FIG. 7: Quantum discord (D) for the Generalized Werner State (i) for $k=0$ as a function of $p$ and $n$ and (ii) for $n=0$ as a function of $p$ and $k$.

S. Takeuchi, Nat. Commun. 4, 2426 (2013); G. H. Low, T. J. Yoder, I. L. Chuang, Phys. Rev. Lett. 114, 100801 (2015).

[5] T. J. Osborne and M. A. Nielsen, Phys. Rev. A 66, 032110 (2002); A. Osterloh et al, Nature 416, 608 (2002); L. Amico et al, Rev. Mod. Phys. 80, 517 (2008); R. Dillenschneider, Phys. Rev. B 78, 224413 (2008); Eisert, J. et al, Rev. Mod. Phys. 82, 277 (2010).

[6] Engel G. S. et al, Nature, 446, 782 (2007); M. Sarovar, et al, Nat. Phys., 6462 (2010).

[7] See, eg, D. Deutsch et al, Phys. Rev. Lett. 77, 2818 (1996); R. Horodecki, M. Horodecki, and P. Horodecki, Phys. Lett. A 222, 21 (1996); Pati, Arun K., Phys. Rev. A, 63 , 014320 (2000); S. Adhikari and B. S. Choudhury, Phys. Rev. A 74, 032323 (2006); V. Madhok and A. Dutta, Phys. Rev. A, 83, 032323 (2011); Sk Sazim and I. Chakrabarty, Eur. Phys. J. D 67, 174 (2013), S. Chatterjee et al, arXiv:1411.4397 (2014).

[8] R. Horodecki, P. Horodecki, M. Horodecki, and K. Horodecki Rev. Mod. Phys., 81, 865, 2009; K. Modi et al., Rev. Mod. Phys., 84, 1655, 2012.

[9] H. Ollivier, W. H. Zurek, Phys. Rev. Lett. 88, 017901 (2001); L. Henderson, V. Vedral, J. Phys. A 34, 6899 (2001); S. Luo, Phys. Rev. A 77, 042303 (2008); K. Modi et al., Rev. Mod. Phys., 84, 1655,2012 and references there in.
[10] M. Horodecki et al., Phys. Rev. A 71, 062307 (2005); A. R. Usha Devi, A. K. Rajagopal, Phys. Rev. Lett. 100, 140502 (2008); K. Modi et al., Phys. Rev. Lett. 104, 080501 (2010); M. Okrasa and Z. Walczak, Europhys. Lett. 96, 60003 (2011); C. C. Rulli. et al., Phys. Rev. A, 84, 042109, (2011).

[11] I. Chakrabarty, P. Agrawal, A. K. Pati, Eur. Phys. J. D 57, 265 (2010).

[12] A.K. Rajagopal and R.W Rendell, Phys. Rev. A, 66, 022104 (2002).

[13] S. Yu, et al, arXiv:1102.4710 (2011); M. Gessner, H.-P. Breuer, Phys. Rev. A, 87, 042107 (2013).

[14] M. Lewenstein, B. Krauss, J. I. Cirac, and P. Horodecki, Phys. Rev. A 62, 052310 (2000).

[15] B. M. Terhal, J. Theor. Comput. Sci. 287, 313 (2002).

[16] J. Sperling and W. Vogel, Phys. Rev. A 79, 022318 (2009).

[17] B. Groisman, S. Popescu, and A. Winter, Phys. Rev. A 72, 032317 (2005); N. Li and S. Lou, Phys. Rev. A 76, 032327 (2007); Z. Walczak, Phys. Lett. A 373, 1818 (2009).

[18] A. Streltsov, H. Kampermann, and D. Bruss, Phys. Rev. Lett. 107, 170502 (2011).

[19] K. Modi, Open Syst. Inf. Dyn., 21, 1440006 (2014).

[20] A. Peres, Phys. Rev. Lett. 771413 (1996); M. Horodecki, P. Horodecki, R. Horodecki, Phys. Lett. A 